Research Paper

\title{
Association between Benzodiazepine Use and Dementia: Data Mining of Different Medical Databases
}

\author{
Mitsutaka Takada ${ }^{凶}$, Mai Fujimoto, Kouichi Hosomi \\ Division of Clinical Drug Informatics, School of Pharmacy, Kinkai University, Higashi-osaka, Osaka, 577-8502, Japan. \\ $\triangle$ Corresponding author: Mitsutaka Takada, PhD. Division of Clinical Drug Informatics, School of Pharmacy, Kindai University, 577-8502, 3-4-1, Kowakae, \\ Higashi-osaka, Osaka, 577-8502, Japan. Telephone number: +81-6-6721-2332 Fax number: +81-6-6730-1394. E-mail address: takada@phar.kindai.ac.jp \\ (C) Ivyspring International Publisher. Reproduction is permitted for personal, noncommercial use, provided that the article is in whole, unmodified, and properly cited. See \\ http://ivyspring.com/terms for terms and conditions.
}

Received: 2016.05.16; Accepted: 2016.09.27; Published: 2016.10.18

\begin{abstract}
Purpose: Some studies have suggested that the use of benzodiazepines in the elderly is associated with an increased risk of dementia. However, this association might be due to confounding by indication and reverse causation. To examine the association between benzodiazepine anxiolytic drug use and the risk of dementia, we conducted data mining of a spontaneous reporting database and a large organized database of prescriptions.

Methods: Data from the US Food and Drug Administration Adverse Event Reporting System (FAERS) from the first quarter of 2004 through the end of 2013 and data from the Canada Vigilance Adverse Reaction Online Database from the first quarter of 1965 through the end of 2013 were used for the analyses. The reporting odds ratio (ROR) and information component (IC) were calculated. In addition, prescription sequence symmetry analysis (PSSA) was performed to identify the risk of dementia after using benzodiazepine anxiolytic drugs over the period of January 2006 to May 2014.

Results: Benzodiazepine use was found to be associated with dementia in analyses using the FAERS database (ROR: 1.63, 95\% Cl: 1.61-1.64; IC: 0.66, 95\% Cl: 0.65-0.67) and the Canada Vigilance Adverse Reaction Online Database (ROR: 1.88, 95\% Cl: 1.83-1.94; IC: 0.85, 95\% Cl: 0.80-0.89). ROR and IC values increased with the duration of action of benzodiazepines. In the PSSA, a significant association was found, with adjusted sequence ratios of 1.24 (1.05-1.45), 1.20 (1.06-1.37), 1.23 (1.11-1.37), 1.34 (1.23-1.47), 1.41 (1.29-1.53), and $1.44(1.33-1.56)$ at intervals of $3,6,12,24,36$, and 48 months, respectively. Furthermore, the additional PSSA, in which patients who initiated a new treatment with benzodiazepines and anti-dementia drugs within 12- and 24-month periods were excluded from the analysis, demonstrated significant associations of benzodiazepine use with dementia risk.

Conclusion: Multi-methodological approaches using different methods, algorithms, and databases suggest that long-term use of benzodiazepines and long-acting benzodiazepines are strongly associated with an increased risk of dementia.
\end{abstract}

Key words:

\section{Introduction}

An increase in the prevalence of dementia has become a serious social problem due to an aging society. Dementia is currently the main cause of dependency in older individuals and a major public health concern affecting approximately 36 million people worldwide. The prevalence of dementia rapidly increases from approximately 2-3\% among individuals aged $70-75$ years to $20-25 \%$ among those aged 85 years or more [1], and the number affected by dementia is expected to double between 2020 and 2040 [2].

Anxiolytics are frequently used for the treatment of mood or anxiety disorders and depression in the elderly in many countries, and benzodiazepines are 
commonly prescribed for this purpose $[3,4]$. However, it is inconclusive as to whether their long-term use is effective and safe. Among the elderly, user prevalence as high as $25 \%$ has been reported [5]. Recently, some studies suggested that the use of benzodiazepines in the elderly is associated with an increased risk of incident cognitive decline or dementia [6-9]. Zhong et al. reported in a meta-analysis of nested case-control or prospective cohort studies that long-term benzodiazepine users have an increased risk of dementia compared with never users [10]. Billioti de Gage et al. systematically reviewed published observational studies assessing the relationship between benzodiazepine use and dementia and concluded that exposure to benzodiazepines was associated with a 1.5- to 2-fold increased risk of dementia [11].

On the other hand, some studies suggested that the observed association between benzodiazepine use and dementia might be due to confounding by indication and reverse causation. Certainly, anxiety can be a prodromal symptom of dementia and is the main indication for prescribing benzodiazepines [12]. Therefore, this association might be considered an early marker of a condition associated with an increased risk of dementia rather than a cause of the dementia itself. Overall, the possibility of an increased risk of dementia is still a matter of debate.

Recently, data mining of medical databases including spontaneous adverse drug reaction databases, claims databases, and prescription databases was reported [13, 14]. Some different methodologies and algorithms have been applied to identify safety signals within these medical databases. The aim of this study was to examine the association between benzodiazepine use and dementia using different methodologies, algorithms, and databases.

\section{Methods}

\section{Spontaneous adverse drug reaction databases}

\section{Data sources}

The US Food and Drug Administration (FDA) Adverse Event Reporting System (FAERS) and the Canada Vigilance Adverse Reaction Online Database are computerized information databases designed to support post-marketing safety surveillance programs for all approved drugs and therapeutic biological products. The FAERS contains all reports of adverse events reported spontaneously by health care professionals, manufacturers, and consumers worldwide. The Canada Vigilance Adverse Reaction Online Database contains all domestic reports of adverse events reported spontaneously by health care professionals, manufacturers, and consumers. The
FAERS consists of seven datasets that include patient demographic and administrative information (file descriptor DEMO), drug and biologic information (DRUG), adverse events (REAC), patient outcomes (OUTC), report sources (RPSR), start and end dates of drug therapy (THER), and indications for use/diagnosis (INDI). A unique number for identifying a FAERS report allows all of the information from different files to be linked. Raw data from the FAERS database can be downloaded freely from the FDA website (http:/ / www.fda.gov/Drugs/ InformationOnDrugs/ucm135151.htm). Data from the first quarter of 2004 through the end of 2013 were included in this study. A total of 4,866,160 reports were obtained. Reports with a common CASE number were identified as duplicate reports, which we excluded from the analyses. Finally, a total of $65,146,507$ drug-reaction pairs were identified from $4,081,582$ reports.

The Canada Vigilance Adverse Reaction Online Database consists of 11 data files that include information on reports and patients (Reports.txt), drug names and their codes (Drug_Product.txt), the active ingredients associated with all drugs (Drug_Product_Ingredients.txt), the reaction terms associated with the report (Reactions.txt), outcome presentation text associated with the outcome code (Outcome_LX.txt), gender presentation text associated with the gender code (Gender_LX.txt), report type presentation text associated with the report type code (Report_Type_LX.txt), report seriousness presentation text associated with the report seriousness code (Seriousness_LX.txt), report source presentation text associated with the report source code (Source_LX.txt), linked/duplicate reports presentation text associated with the code (Report_Links_LX.txt), drugs associated with specific reports (Report_Drug.txt), and indications associated with specific reports (Report_Drug_Indication.txt). Raw data from the Canada Vigilance Adverse Reaction Online Database can be downloaded freely from the Health Canada website (http://www.hc-sc.gc.ca/dhp-mps/medeff/databas don/index-eng.php). Data from the first quarter of 1965 through the end of 2013 were included in this study. A total of 4,019,652 drug-reaction pairs were identified from 381,822 reports.

The Medical Dictionary for Regulatory Activities (MedDRA ${ }^{\circledR}$ version 17.0) preferred terms (PTs) were used to classify the adverse events.

\section{Identification of benzodiazepine anxiolytic drugs}

Benzodiazepines with the World Health Organization Anatomical and Therapeutic Chemical 
code of N05C were defined as benzodiazepine anxiolytic drugs in this study (Table 1). A drug name archive that included the names of all preparations, generic names, and drug synonyms marketed worldwide was created using the Martindale website (https://www.medicinescomplete.com/mc/login.ht $\mathrm{m})$. Benzodiazepine anxiolytic drugs were identified by linking this archive with the FAERS database and the Canada Vigilance Adverse Reaction Online Database (Table 1). All records including benzodiazepine anxiolytic drugs were selected, and the relevant reactions were then identified.

Table 1. Benzodiazepine anxiolytic drugs.

\begin{tabular}{ll}
\hline Short-acting & Etizolam \\
& Clotiazepam \\
& Flutazolam \\
Medium-acting & Lorazepam \\
& Alprazolam \\
& Fludiazepam \\
& Bromazepam \\
& Nimetazepam \\
Long-acting & Diazepam \\
& Cloxazolam \\
& Chlordiazepoxide \\
& Medazepam \\
& Clorazepate \\
Ultra-long-acting & Flutoprazepam \\
& Loflazepate \\
& Mexazolam \\
& Oxazolam \\
& Prazepam \\
\hline
\end{tabular}

\section{Definition of adverse events}

Adverse events in the FAERS and the Canada Vigilance Adverse Reaction Online Database were coded using the MedDRA® PTs, which are categorized by defined medical condition or area of interest. Using the Standardized MedDRA $®$ Queries, we identified 82 PTs in the category of dementia.

\section{Statistical analysis}

The reporting odds ratio (ROR) [15] and the information component (IC) [16] were used to detect spontaneous report signals. These signal scores were calculated using a case/non-case method [17, 18]. Cases are the reports involving the event of interest (i.e., dementia) and the non-cases all other reports. ROR and IC are widely used and employed by the Netherlands Pharmacovigilance Centre and the World Health Organization, respectively $[15,16]$. All of these algorithms were used to calculate signal scores to assess whether a drug was significantly associated with an adverse event or not from a two-by-two frequency table of counts. However, these calculations or algorithms, so-called disproportionality analyses or measures, differ from one another in that the ROR is frequentist (non-Bayesian), whereas the IC is Bayesian. For the ROR, a signal is detected if the lower limit of the $95 \%$ two-sided confidence interval $(95 \% \mathrm{CI})$ is $>1$ [15]. For the IC, a signal is detected if the IC025 metric, the lower limit of the $95 \% \mathrm{CI}$ of the IC, is $>0$ [16]. In the current study, these two methods were used for signal detection, and the adverse events were considered to be drug-associated if the two indices met the criteria indicated above. Data management and analyses were performed using Visual Mining Studio software (version 8.1; Mathematical Systems, Inc. Tokyo, Japan).

\section{Prescription sequence symmetry analysis (PSSA)}

\section{Data source}

A large organized database of prescriptions constructed by a database vendor (Japan Medical Information Research Institute, Inc. Japan [JMIRI]) was used in the study. The JMIRI prescription database consists of prescriptions collected from approximately 700 pharmacies in Japan. The database includes approximately 92,700,000 prescriptions for approximately 10,300,000 patients from January 2006 to May 2014. For the PSSA, we identified cases extracted from this database for whom benzodiazepine anxiolytic drugs (Table 1) and anti-dementia drugs (donepezil, galantamine, rivastigmine, and memantine) were prescribed at least once during the study period. The data included encrypted personal identifiers: month/year of birth and gender of the patient, drug name, unique drug code and generic name, and prescribing date. Data on drugs dispensed in hospitals were not included. This study was approved by the Ethics Committee of Kindai University School of Pharmacy.

\section{Study design}

The PSSA was performed to test the hypothesis that benzodiazepines increase the risk of dementia. The PSSA method has been described in detail in several published studies investigating the associations between the use of certain target drugs and potential adverse events $[19,20]$. Briefly, the PSSA evaluates asymmetry in the distribution of an incident event (e.g., prescription of another drug) before and after the initiation of a specific treatment. Asymmetry may indicate an association between the specific treatment of interest and the event. PSSA is based on a situation in which drug A is suspected of causing an adverse event that itself is treated by drug $B$ [19]. In this study, anti-dementia drugs were used as markers of dementia caused by benzodiazepines, and 
the association between benzodiazepines and anti-dementia drug uses was analyzed.

The ratio of the numbers of patients with a prescription for anti-dementia drugs after versus before the initiation of benzodiazepines was defined as the crude sequence ratio (SR). A SR $>1$ indicated an increased risk of benzodiazepine-induced dementia. The SR is sensitive to prescribing trends over time. Therefore, the SRs were adjusted for temporal trends in benzodiazepines and anti-dementia drugs using the method proposed by Halls [19]. The probability that benzodiazepines were prescribed first, in the absence of any causal relationship, can be estimated by the so-called null-effect SR [19]. The null-effect SR yielded by the proposed model may be interpreted as a reference value for the SR. Therefore, the null-effect SR is the expected SR in the absence of any causal association, after taking the incidence trends into account. By dividing the crude SR by the null-effect SR, an adjusted SR (ASR) corrected for temporal trends is obtained. A slightly modified model was used to account for the limited time interval allowed between the benzodiazepine prescription and treatment of dementia [20]. The major advantage of the SR is that it is robust for confounders that are stable over time. Significant confounding factors including age, gender, and frequency of visits did not cause an asymmetrical distribution of benzodiazepines and anti-dementia drugs [20].

\section{Data analysis}

PSSA was undertaken to identify the new use of anti-dementia drugs, which served as a marker of benzodiazepine-induced dementia. All incident users of benzodiazepines and anti-dementia drugs were identified from January 2006 to May 2014. In this study, patients included in the database were followed up until May 2014, and therefore different patients had different follow-up periods. Incidence was defined as the first prescription of target drugs. To exclude prevalent users of target drugs, the analysis was restricted to users who presented their first prescription on July 2006 or later, that is, after a run-in period of 6 months. To ensure that our analysis was restricted to incident users, we also performed a waiting time distribution analysis [21]. An identical run-in period was also applied to patients enrolled in the cohort after July 2006 to exclude prevalent users of the target drugs. The analysis was based on the principle that by observing the first occurrence of a prescription within a specific time window, prevalent users of the drug will cluster at the beginning of the observation period when the prescription is repeated within a short time period. In contrast, incident users will be distributed evenly throughout the observation period. Incident users were identified by excluding those patients who had received their first prescription for the target drugs prior to June 2006. All patients were identified who initiated new treatments with both benzodiazepines and anti-dementia drugs within 3-, 6-, 12-, 24-, 36- and 48-month periods. Patients who had received their first prescriptions for benzodiazepines and anti-dementia drugs on the same date were not included in the determination of the SR.

Results are expressed as the means \pm standard deviation (SD) for quantitative data and as frequencies (percentage) for categorical data. The 95\% CIs for the ASRs were calculated using a method for exact CIs for binomial distributions [22].

\section{Additional analysis}

The additional PSSA was performed in order to address the problem about confounding by indication and reverse causation bias. If indication and reverse causation bias is responsible for the association between benzodiazepine use and dementia, the association for recent users should be stronger than that for past users [23]. Therefore, patients who initiated a new treatment with benzodiazepines and anti-dementia drugs within 12- and 24-month periods were excluded from the analysis to minimize the effect of this confounding.

\section{Results}

\section{FAERS database analyses}

A total of 1,971,750 drug-reaction pairs for dementia were found in the FAERS database. The statistical results from the FAERS database analyses are presented in Table 2 . The signal scores suggested that benzodiazepines were associated with dementia (ROR: 1.63, 95\% CI: 1.61-1.64; IC: 0.66, 95\% CI: 0.65-0.67). In addition, classes of short-, medium-, long-, and ultra-long-acting benzodiazepines were also associated with dementia. The ROR and IC values increased with the duration of benzodiazepine action (Figure 1A). In the analyses of individual benzodiazepines, significant associations with dementia were found for etizolam, clotiazepam, lorazepam, alprazolam, bromazepam, diazepam, cloxazolam, chlordiazepoxide, medazepam, clorazepate, loflazepate, oxazolam, and prazepam.

\section{Canada Vigilance Adverse Reaction Online Database}

A total of 141,451 drug-reaction pairs for dementia were found in the Canada Vigilance Adverse Reaction Online Database. The statistical results from the analyses using this database are presented in Table 3 . The signal scores suggested that 
a class of benzodiazepines is associated with dementia (ROR: 1.88, 95\% CI: 1.83-1.94; IC: 0.85, 95\% CI: 0.80-0.89). In addition, medium- and long-acting benzodiazepines were also associated with dementia. The ROR and IC values of long-acting benzodiazepines were higher than those of medium-acting benzodiazepines (Figure 1B). In the analyses of individual benzodiazepines, significant associations with dementia were found for lorazepam, bromazepam, diazepam, chlordiazepoxide, and clorazepate.

\section{PSSA}

The characteristics of the study population of the PSSA are summarized in Table 4. The number of prescriptions including those for benzodiazepines during the study period was $9,332,070$. Of the 577,099 benzodiazepine users, 145,496 incident users were identified. The mean age of the incident users was $59.5 \pm 18.7$ years.
Table 2. The association between individual benzodiazepine use and dementia based on the FAERS database.

\begin{tabular}{|c|c|c|c|c|c|c|}
\hline & Cases & Non-cases & ROR & $95 \% \mathrm{CI}$ & IC & $95 \% \mathrm{CI}$ \\
\hline Benzodiazepines & 50,302 & $1,001,070$ & 1.63 & $1.61-1.64$ & 0.66 & $0.65-0.67$ \\
\hline Short-acting & 800 & 18,637 & 1.38 & $1.28-1.48$ & 0.44 & $0.34-0.55$ \\
\hline Etizolam & 691 & 16,019 & 1.38 & $1.28-1.49$ & 0.45 & $0.34-0.56$ \\
\hline Clotiazepam & 107 & 2,597 & 1.32 & $1.09-1.60$ & 0.38 & $0.10-0.67$ \\
\hline Flutazolam & 2 & 21 & 3.05 & $0.72-13.01$ & 0.80 & $-0.97-2.56$ \\
\hline Medium-acting & 35,772 & 739 & 1.56 & $1.54-1.58$ & 0.61 & $0.59-0.62$ \\
\hline Lorazepam & 16,245 & 327959 & 1.59 & $1.57-1.62$ & 0.64 & $0.62-0.66$ \\
\hline Alprazolam & 18,075 & 384,519 & 1.51 & $1.49-1.53$ & 0.57 & $0.55-0.59$ \\
\hline Fludiazepam & 3 & 279 & 0.34 & $0.11-1.07$ & -1.26 & $-2.71-0.20$ \\
\hline Bromazepam & 1,444 & 26,161 & 1.77 & $1.68-1.87$ & 0.79 & $0.71-0.87$ \\
\hline Nimetazepam & 5 & 98 & 1.63 & $0.67-4.02$ & 0.53 & $-0.68-1.74$ \\
\hline Long-acting & 13,186 & 233,750 & 1.81 & $1.78-1.85$ & 0.82 & $0.79-0.84$ \\
\hline Diazepam & 11,267 & 195,417 & 1.85 & $1.82-1.89$ & 0.85 & $0.82-0.88$ \\
\hline Cloxazolam & 200 & 3,084 & 2.08 & $1.80-2.40$ & 1.00 & $0.79-1.21$ \\
\hline Chlordiazepoxide & 740 & 17,880 & 1.33 & $1.23-1.43$ & 0.39 & $0.28-0.50$ \\
\hline Medazepam & 15 & 220 & 2.18 & $1.29-3.69$ & 0.97 & $0.23-1.72$ \\
\hline Clorazepate & 964 & 17,149 & 1.80 & $1.69-1.92$ & 0.81 & $0.72-0.91$ \\
\hline Ultra-long-acting & 544 & 9,667 & 1.80 & $1.65-1.97$ & 0.81 & $0.69-0.94$ \\
\hline Flutoprazepam & 6 & 161 & 1.19 & $0.53-2.70$ & 0.20 & $-0.91-1.32$ \\
\hline Loflazepate & 185 & 3,483 & 1.70 & $1.47-1.97$ & 0.73 & $0.51-0.95$ \\
\hline Mexazolam & 7 & 182 & 1.23 & $0.58-2.62$ & 0.24 & $-0.80-1.29$ \\
\hline Oxazolam & 18 & 294 & 1.96 & $1.22-3.16$ & 0.86 & $0.18-1.54$ \\
\hline
\end{tabular}

Cases: number of reports of dementia

Non-cases: all reports of adverse drug reactions other than dementia ROR: reporting odds ratio

IC: information component

CI: confidence interval
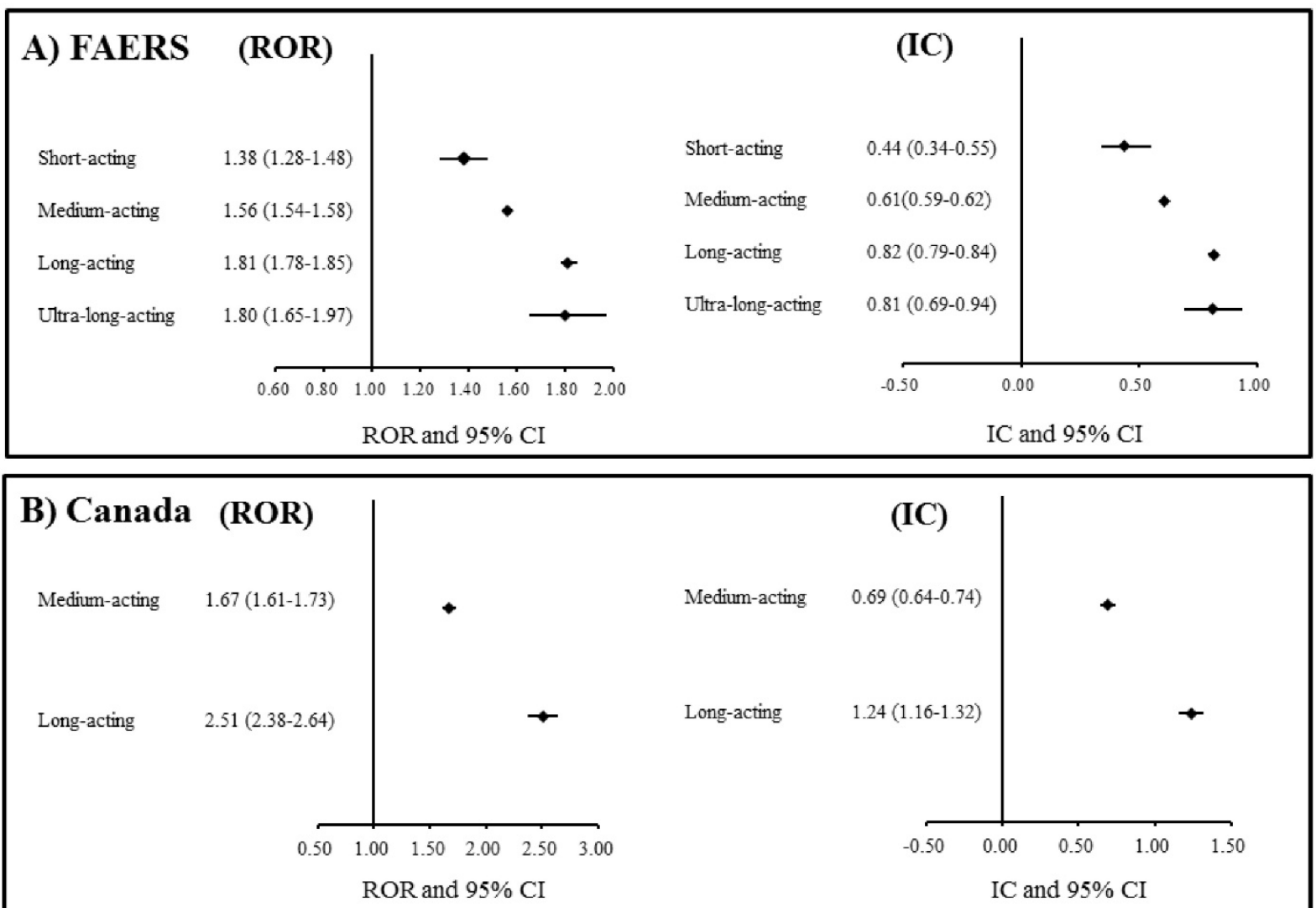

Figure 1. The associations between benzodiazepines and dementia in the analysis of the spontaneous adverse drug reaction databases. A) FAERS: The US Food and Drug Administration (FDA) Adverse Event Reporting System. B) Canada: Canada Vigilance Adverse Reaction Online Database. ROR: Reporting odds ratio. Cl: Confidence interval. IC: Information component. 
The associations between benzodiazepine and anti-dementia drug use are shown in Table 5. Of the 145,496 incident users of benzodiazepines, 3,384 were also identified as incident users of anti-dementia drugs, before or after the initiation of the benzodiazepines. A significant association between benzodiazepine and anti-dementia drug use was found, with ASRs of 1.24 (1.05-1.45), 1.20 (1.06-1.37), 1.23 (1.11-1.37), 1.34 (1.23-1.47), 1.41 (1.29-1.53), and 1.44 (1.33-1.56) at intervals of $3,6,12,24,36$, and 48 months, respectively. Interval-related change in the ASRs of benzodiazepines and individual classes of benzodiazepines are presented in Figure 2. The ASRs of benzodiazepines increased with the interval duration. The ASRs of long- and ultra-long-acting benzodiazepines were higher than those of short- and medium-acting benzodiazepines for the relatively short intervals. However, at the 48-month interval, remarkable differences were not observed among the individual classes of benzodiazepines, and all classes were associated with dementia.

A significant association between benzodiazepine and anti-dementia drug use was also found in the additional PSSA (Table 6A, Table 6B). Exclusion of the patients who initiated a new treatment with benzodiazepines and anti-dementia drugs within 12- and 24-month periods did not affect the risk of dementia induced by benzodiazepines.

A summary of the signals detected for benzodiazepine-associated dementia is presented in Table 7.
Table 3. The association between individual benzodiazepine use and dementia based on the Canada Vigilance Adverse Reaction Online Database

\begin{tabular}{|c|c|c|c|c|c|c|}
\hline & Cases & Non-cases & ROR & $95 \% \mathrm{CI}$ & IC & $95 \% \mathrm{CI}$ \\
\hline Benzodiazepines & 4,877 & 72,164 & 1.88 & $1.83-1.94$ & 0.85 & $0.80-0.89$ \\
\hline Short-acting & - & - & - & - & - & - \\
\hline Medium-acting & 3,317 & 54979 & 1.67 & $1.61-1.73$ & 0.69 & $0.64-0.74$ \\
\hline Lorazepam & 2,818 & 41,481 & 1.88 & $1.81-1.95$ & 0.85 & $0.80-0.91$ \\
\hline Alprazolam & 404 & 11,810 & 0.94 & $0.85-1.04$ & -0.09 & $-0.24-0.06$ \\
\hline Bromazepam & 95 & 1,688 & 1.54 & $1.26-1.90$ & 0.59 & $0.29-0.89$ \\
\hline Long-acting & 1,560 & 17,185 & 2.51 & $2.38-2.64$ & 1.24 & $1.16-1.32$ \\
\hline Diazepam & 1,268 & 14,463 & 2.42 & $2.28-2.56$ & 1.19 & $1.11-1.28$ \\
\hline Chlordiazepoxide & 256 & 2,360 & 2.98 & $2.62-3.39$ & 1.47 & $1.28-1.65$ \\
\hline Medazepam & 0 & 10 & 0.00 & - & -0.47 & $-3.49-2.54$ \\
\hline Clorazepate & 36 & 352 & 2.80 & $1.99-3.95$ & 1.33 & $0.84-1.83$ \\
\hline Ultra-long-acting & - & - & - & - & - & - \\
\hline
\end{tabular}

Cases: number of reports of dementia

Non-cases: all reports of adverse drug reactions other than dementia

ROR: reporting odds ratio

IC: information component

CI: confidence interval

Table 4. Characteristics of the study population in the prescription sequence symmetry analysis

\begin{tabular}{llll}
\hline & Total & Male & Female \\
\hline $\begin{array}{l}\text { Benzodiazepine prescriptions, } \mathrm{n} \\
\text { Users }\end{array}$ & $5,332,070$ & & \\
Incident users, $\mathrm{n}$ & 145,099 & & \\
Age, years, $\mathrm{n}(\%)$ & & $49,972(34.3)$ & $95,524(65.7)$ \\
$<20$ & $3,202(2.2)$ & $1,350(2.7)$ & $1,852(1.9)$ \\
$20-39$ & $22,550(15.5)$ & $7,607(15.2)$ & $14,943(15.6)$ \\
$40-59$ & $37,253(25.6)$ & $13,327(25.6)$ & $23,926(25.0)$ \\
$60-79$ & $63,207(43.4)$ & $21,706(43.4)$ & $41,501(43.4)$ \\
$\geq 80$ & $19,284(13.3)$ & $5,982(13.3)$ & $13,302(12.0)$ \\
Mean \pm SD & $59.5 \pm 18.7$ & $58.9 \pm 18.6$ & $59.8 \pm 18.7$ \\
\hline SD: standard deviation & & &
\end{tabular}

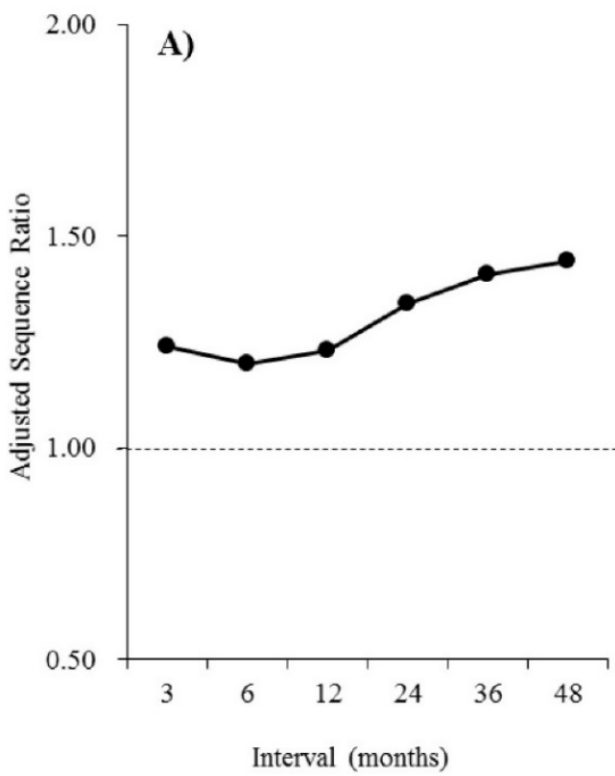

Figure 2. Interval-related changes in the adjusted sequence ratios of benzodiazepines. A) Benzodiazepines. B) Individual classes of benzodiazepines. 
Table 5. Prescription sequence symmetry analysis: associations of benzodiazepine use with dementia

\begin{tabular}{|c|c|c|c|c|c|c|c|c|}
\hline & \multirow{3}{*}{$\begin{array}{l}\text { Incident } \\
\text { users }\end{array}$} & \multirow{3}{*}{$\begin{array}{l}\text { Concomitant use with } \\
\text { anti-dementia drugs }\end{array}$} & \multirow{3}{*}{$\begin{array}{l}\text { Interval } \\
\text { (months) }\end{array}$} & \multirow{2}{*}{\multicolumn{2}{|c|}{$\begin{array}{l}\text { No. of patients prescribed } \\
\text { anti-dementia drugs }\end{array}$}} & \multirow[t]{3}{*}{ Adjusted SR } & \multicolumn{2}{|c|}{$95 \% \mathrm{CI}$} \\
\hline & & & & & & & \multirow{2}{*}{ Lower } & \multirow[t]{2}{*}{ Upper } \\
\hline & & & & last & first & & & \\
\hline \multirow[t]{6}{*}{ Benzodiazepines } & 145,496 & 3,384 & 3 & 348 & 277 & 1.24 & 1.05 & 1.45 \\
\hline & & & 6 & 539 & 434 & 1.20 & 1.06 & 1.37 \\
\hline & & & 12 & 821 & 629 & 1.23 & 1.11 & 1.37 \\
\hline & & & 24 & 1,233 & 816 & 1.34 & 1.23 & 1.47 \\
\hline & & & 36 & 1,514 & 894 & 1.41 & 1.29 & 1.53 \\
\hline & & & 48 & 1,718 & 935 & 1.44 & 1.33 & 1.56 \\
\hline \multirow[t]{6}{*}{ Short-acting } & 107,866 & 2,563 & 3 & 242 & 203 & 1.17 & 0.97 & 1.42 \\
\hline & & & 6 & 399 & 326 & 1.18 & 1.02 & 1.38 \\
\hline & & & 12 & 607 & 475 & 1.20 & 1.06 & 1.36 \\
\hline & & & 24 & 924 & 617 & 1.32 & 1.19 & 1.46 \\
\hline & & & 36 & 1,159 & 672 & 1.42 & 1.29 & 1.56 \\
\hline & & & 48 & 1,310 & 703 & 1.44 & 1.32 & 1.58 \\
\hline \multirow[t]{6}{*}{ Medium-acting } & 41,870 & 1,082 & 3 & 106 & 105 & 1.00 & 0.75 & 1.32 \\
\hline & & & 6 & 158 & 164 & 0.94 & 0.75 & 1.17 \\
\hline & & & 12 & 252 & 231 & 1.03 & 0.86 & 1.24 \\
\hline & & & 24 & 371 & 295 & 1.13 & 0.97 & 1.32 \\
\hline & & & 36 & 457 & 336 & 1.15 & 1.00 & 1.33 \\
\hline & & & 48 & 522 & 353 & 1.18 & 1.03 & 1.35 \\
\hline \multirow[t]{6}{*}{ Long-acting } & 31,698 & 773 & 3 & 83 & 52 & 1.57 & 1.09 & 2.26 \\
\hline & & & 6 & 124 & 88 & 1.36 & 1.02 & 1.80 \\
\hline & & & 12 & 181 & 127 & 1.32 & 1.05 & 1.67 \\
\hline & & & 24 & 272 & 180 & 1.31 & 1.08 & 1.59 \\
\hline & & & 36 & 340 & 197 & 1.39 & 1.16 & 1.66 \\
\hline & & & 48 & 401 & 214 & 1.42 & 1.20 & 1.68 \\
\hline \multirow[t]{6}{*}{ Ultra-long-acting } & 20,492 & 492 & 3 & 64 & 35 & 1.80 & 1.17 & 2.80 \\
\hline & & & 6 & 103 & 58 & 1.71 & 1.23 & 2.41 \\
\hline & & & 12 & 146 & 90 & 1.51 & 1.15 & 1.99 \\
\hline & & & 24 & 206 & 118 & 1.51 & 1.20 & 1.91 \\
\hline & & & 36 & 242 & 135 & 1.44 & 1.16 & 1.79 \\
\hline & & & 48 & 264 & 141 & 1.41 & 1.15 & 1.75 \\
\hline
\end{tabular}

Incident users: number of patients who received their first prescription for benzodiazepines

No. of patients prescribed anti-dementia drugs last: the number of patients prescribed anti-dementia drugs after benzodiazepine use

No. of patients prescribed anti-dementia drugs last: the number of patients prescribed anti-dementia drugs before benzodiazepine use

Adjusted SR: adjusted sequence ratio

CI: confidence interval

Table 6A. Additional prescription sequence symmetry analysis. Patients who initiated a new treatment with benzodiazepines and anti-dementia drugs within 12-month period were excluded from the analysis.

\begin{tabular}{|c|c|c|c|c|c|c|}
\hline & \multirow{3}{*}{$\begin{array}{c}\text { Interval } \\
\text { (months) }\end{array}$} & \multirow{2}{*}{\multicolumn{2}{|c|}{$\begin{array}{l}\text { No. of patients prescribed } \\
\text { anti-dementia drugs }\end{array}$}} & \multirow[t]{3}{*}{ Adjusted SR } & \multicolumn{2}{|c|}{$95 \% \mathrm{CI}$} \\
\hline & & & & & \multirow[t]{2}{*}{ Lower } & \multirow[t]{2}{*}{ Upper } \\
\hline & & Last & first & & & \\
\hline \multirow[t]{3}{*}{ Benzodiazepines } & 24 & 412 & 187 & 1.96 & 1.64 & 2.34 \\
\hline & 36 & 693 & 265 & 2.17 & 1.88 & 2.51 \\
\hline & 48 & 897 & 306 & 2.30 & 2.01 & 2.62 \\
\hline
\end{tabular}

Incident users: number of patients who received their first prescription for benzodiazepines

No. of patients prescribed anti-dementia drugs last: the number of patients prescribed anti-dementia drugs after benzodiazepine use

No. of patients prescribed anti-dementia drugs last: the number of patients prescribed anti-dementia drugs before benzodiazepine use

Adjusted SR: adjusted sequence ratio

CI: confidence interval

Table 6B. Additional prescription sequence symmetry analysis. Patients who initiated a new treatment with benzodiazepines and anti-dementia drugs within 24-month period were excluded from the analysis.

\begin{tabular}{|c|c|c|c|c|c|c|}
\hline & \multirow{3}{*}{$\begin{array}{l}\text { Interval } \\
\text { (months) }\end{array}$} & \multirow{2}{*}{\multicolumn{2}{|c|}{$\begin{array}{l}\text { No. of patients prescribed } \\
\text { anti-dementia drugs }\end{array}$}} & \multirow[t]{3}{*}{ Adjusted SR } & \multicolumn{2}{|c|}{$95 \%$ CI } \\
\hline & & & & & \multirow[t]{2}{*}{ Lower } & \multirow[t]{2}{*}{ Upper } \\
\hline & & Last & first & & & \\
\hline \multirow[t]{2}{*}{ Benzodiazepines } & 36 & 281 & 78 & 2.99 & 2.32 & 3.90 \\
\hline & 48 & 485 & 119 & 3.19 & 2.61 & 3.94 \\
\hline
\end{tabular}

Incident users: number of patients who received their first prescription for benzodiazepines

No. of patients prescribed anti-dementia drugs last: the number of patients prescribed anti-dementia drugs after benzodiazepine use

No. of patients prescribed anti-dementia drugs last: the number of patients prescribed anti-dementia drugs before benzodiazepine use

Adjusted SR: adjusted sequence ratio

CI: confidence interval 
Table 7. Summary of signal detection for benzodiazepine-associated dementia

\begin{tabular}{|c|c|c|c|}
\hline & FAERS & Canada & Prescription \\
\hline Benzodiazepines & $\uparrow$ & $\uparrow$ & $\uparrow$ \\
\hline Short-acting & $\uparrow$ & - & $\uparrow$ \\
\hline Etizolam & $\uparrow$ & - & $\uparrow$ \\
\hline Clotiazepam & $\uparrow$ & - & $\uparrow$ \\
\hline Flutazolam & nd & - & nd \\
\hline Medium-acting & $\uparrow$ & $\uparrow$ & $\uparrow$ \\
\hline Lorazepam & $\uparrow$ & $\uparrow$ & nd \\
\hline Alprazolam & $\uparrow$ & nd & nd \\
\hline Fludiazepam & nd & - & nd \\
\hline Bromazepam & $\uparrow$ & $\uparrow$ & $\uparrow$ \\
\hline Nimetazepam & nd & - & nd \\
\hline Long-acting & $\uparrow$ & $\uparrow$ & $\uparrow$ \\
\hline Diazepam & $\uparrow$ & $\uparrow$ & $\uparrow$ \\
\hline Cloxazolam & $\uparrow$ & - & $\uparrow$ \\
\hline Chlordiazepoxide & $\uparrow$ & $\uparrow$ & $\uparrow$ \\
\hline Medazepam & $\uparrow$ & - & nd \\
\hline Clorazepate & $\uparrow$ & $\uparrow$ & nd \\
\hline Ultra-long-acting & $\uparrow$ & - & $\uparrow$ \\
\hline Flutoprazepam & nd & - & nd \\
\hline Loflazepate & $\uparrow$ & - & $\uparrow$ \\
\hline Mexazolam & nd & - & nd \\
\hline Oxazolam & $\uparrow$ & - & nd \\
\hline Prazepam & $\uparrow$ & - & - \\
\hline \multicolumn{4}{|c|}{$\begin{array}{l}\text { FAERS: The US Food and Drug Administration (FDA) Adverse Event Reporting } \\
\text { System }\end{array}$} \\
\hline
\end{tabular}

\section{Discussion}

This study suggested that benzodiazepine anxiolytic drug use is associated with the development of dementia. In the analyses of short-, medium-, long-, and ultra-long-acting benzodiazepines, all classes of benzodiazepines are associated with an increased risk of dementia. In the analysis of the FAERS database, the ROR and IC values increased with the duration of benzodiazepine action. In the Canada Vigilance Adverse Reaction Online Database, the ROR and IC values of long-acting benzodiazepines were higher than those of medium-acting benzodiazepines. In the PSSA, ASRs of ultra-long- and long-acting benzodiazepines were higher than those of short- and medium-acting benzodiazepines at relatively short intervals. Given these considerations, it is reasonable to assume that the strength of the association between benzodiazepine use and dementia was stronger for long-acting than for short-acting benzodiazepines. This finding is consistent with a previously reported case-control study investigating the relationship between the risk of Alzheimer's disease and exposure to benzodiazepines [24]. In addition, the ASR of benzodiazepines increased with the interval duration, suggesting that long-term use of benzodiazepines might be associated with an increased risk of dementia. Although we did not directly investigate the relationships between the benzodiazepine cumulative dose and treatment duration and the risk of dementia, our findings obtained from the PSSA supported the hypothesis that long-term use of benzodiazepines was associated with an increased risk of dementia.

The association between benzodiazepine use and dementia might be an early marker of a condition associated with an increased risk of dementia rather than a cause of the dementia itself. Some studies suggested that the frequency of symptoms is highly correlated with an increase in benzodiazepine prescriptions during the years preceding a diagnosis of dementia [12, 25, 26]. Therefore, the observed association between benzodiazepine use and dementia may be due to confounding by indication and reverse causation. If reverse causation is responsible for the association between benzodiazepine use and dementia, the association for recent users should be stronger than that for past users. Imfeld et al. reported increased risks of developing Alzheimer's disease and vascular dementia in those who started benzodiazepines $<1$ year before diagnosis, but not for those who started them 2-4 years before diagnosis [23]. On the other hand, Zhong et al. recently reported in a meta-analysis that long-term benzodiazepine users have an increased risk of dementia compared with never users [7], attenuating the likelihood that reverse causation plays a role. In our study, the risk of dementia for benzodiazepines increased with the interval duration in the PSSA. Furthermore, the additional PSSA, in which patients who initiated a new treatment with benzodiazepines close to the prescription of anti-dementia drugs were excluded from the analysis, also demonstrated significant associations of benzodiazepine use with dementia risk. These findings suggested that confounding by indication and reverse causation bias may not have a serious impact on the study. However, from the perspective of drug action duration, ultra-long- and long-acting benzodiazepines showed declining risks with an increasing interval duration, suggesting that reverse causation might play a role in some cases during the early phase of treatment. Although this finding might support the likelihood of reverse causation having an influence, significantly increased risks were observed for all classes of benzodiazepines at the 48-month interval. Therefore, it is reasonable to consider that long-term use of benzodiazepines is associated with an increased risk of dementia. 
Analysis of spontaneous reporting databases is a useful method for identifying risk signals; however, there are several potential limitations that should be taken into account when interpreting the results. First, there is no certainty that the reported event was actually due to the drug. Second, not every adverse event or medication error associated with a drug product gets reported to the regulating authorities. Third, no individual database or algorithm has been found to be sufficient for signal detection. Therefore, a different methodology, PSSA of the JMIRI prescription database, was used to confirm the findings from spontaneous reporting databases. Of course, there are several potential limitations associated with such an analysis based on a database of prescriptions collected from pharmacies. First, anti-Alzheimer's disease drugs were used as surrogate markers for dementia. Therefore, to be precise, the association between benzodiazepine use and Alzheimer's disease was evaluated in the analysis of the JMIRI database. In addition, individual cases were not reviewed, and other causes were not considered; therefore, some patients may not in fact have had dementia. Conversely, the PSSA is similar to the case-crossover design, in which exposures during a fixed period before the case date (date when the target outcome occurred) are compared with certain prior dates in the same individual [27]. These within-subject comparisons can thus be fully controlled for potential confounding due to between-subject differences and time-invariant characteristics, e.g., age, gender, genetic factors, mental health status, and other unknown confounding factors. PSSA has been employed in a number of previous studies investigating the associations between use of certain target drugs and potential adverse events [13, 14, 19, 20, 28], and the validity of the PSSA has been confirmed by previous studies $[29,30]$.

Although a plausible pharmacological mechanism for dementia is unknown, several theories have been reported. Benzodiazepines have deleterious effects on memory, and benzodiazepine use induces both non-amnestic and amnestic mild cognitive impairment [31, 32]. This effect may precipitate dementia progression [33, 34]. Long-term administration of benzodiazepines down-regulates the levels of their binding receptors [35], resulting in a cognitive decline [36]. We found that benzodiazepine use, particularly long-acting drugs, might be associated with increased risks of dementia. If these observed associations are causal, benzodiazepines should be used as briefly as possible to reduce possible adverse reactions.

\section{Conclusions}

The results of a multi-methodological approach, using different methods, algorithms, and databases, suggest that benzodiazepine use, especially long-term use and long-acting drugs, is associated with an increased risk of dementia. Individuals prescribed benzodiazepines should be considered to have an increased risk of dementia. Although the biological mechanism for this phenomenon remains unknown, the risk of dementia associated with benzodiazepine use is a very important finding in clinical practice. Patients using benzodiazepines should be closely monitored for the development of dementia in clinical practice, and further studies are needed to confirm our findings and elucidate the mechanisms underlying benzodiazepine-induced dementia.

\section{Abbreviations}

FAERS: FDA Adverse Event Reporting System; FDA: Food and Drug Administration; MedDRA: Medical Dictionary for Regulatory Activities; PT: preferred term; ROR: reporting odds ratio; IC: information component; JMIRI: Japan Medical Information Research Institute; ICD-10: International Classification of Disease, 10th Revision; PSSA: Prescription sequence symmetry analysis; SR: Sequence ratio.

\section{Acknowledgements}

We thank the Japan Medical Information Research Institute, Inc. for providing the database of prescriptions.

\section{Competing Interests}

The authors have declared that no competing interest exists.

\section{References}

1. Ferri CP, Prince M, Brayne C, et al. Global prevalence of dementia: a Delphi consensus study. Lancet. 2005; 366: 2112-2117.

2. Rizzi L, Rosset I, Roriz-Cruz M. Global epidemiology of dementia: Alzheimer's and vascular types. Biomed Res Int. 2014; 2014: 908915.

3. Linden M, Bar T, Helmchen H. Prevalence and appropriateness of psychotropic drug use in old age: results from the Berlin Aging Study (BASE). Int Psychogeriatr. 2004; 16: 461-480.

4. Ashton $\mathrm{H}$. The diagnosis and management of benzodiazepine dependence. Curr Opin Psychiatry. 2005; 18: 249-255.

5. Hogan DB, Maxwell CJ, Fung TS, et al. Prevalence and potential consequences of benzodiazepine use in senior citizens: results from the Canadian Study of Health and Aging. Can J Clin Pharmacol. 2003; 10: 72-77.

6. Curran HV, Collins R, Fletcher $S$, et al. Older adults and withdrawal from benzodiazepine hypnotics in general practice: effects on cognitive function, sleep, mood and quality of life. Psychol Med. 2003; 33: 1223-1237.

7. Zhong G, Wang Y, Zhang Y, et al. Association between benzodiazepine use and dementia: A Meta-Analysis. PLoS One. 2015; 10: e0127836.

8. Gallacher J, Elwood P, Pickering J, et al. Benzodiazepine use and risk of dementia: evidence from the Caerphilly Prospective Study (CaPS). J Epidemiol Community Health. 2012; 66: 869-873.

9. Lagnaoui R, Begaud B, Moore N, et al. Benzodiazepine use and risk of dementia: a nested case-control study. J Clin Epidemiol. 2002; 55: 314-318.

10. Zhou Y, Yuan Y, Cai RR, et al. Statin therapy on glycaemic control in type 2 diabetes: a meta-analysis. Expert Opin Pharmacother. 2013; 14: 1575-1584. 
11. Billioti de Gage S, Pariente A, Begaud B. Is there really a link between benzodiazepine use and the risk of dementia? Expert Opin Drug Saf. 2015; 14: 733-747.

12. Amieva $H$, Le Goff $M$, Millet $X$, et al. Prodromal Alzheimer's disease: successive emergence of the clinical symptoms. Ann Neurol. 2008; 64: 492-498.

13. Takada M, Fujimoto M, Yamazaki K, et al. Association of statin use with sleep disturbances: data mining of a spontaneous reporting database and a prescription database. Drug Saf. 2014; 37: 421-431.

14. Fujimoto M, Higuchi T, Hosomi K, et al. Association between statin use and cancer: data mining of a spontaneous reporting database and a claims database. Int J Med Sci. 2015; 12: 223-233.

15. van Puijenbroek EP, Bate A, Leufkens HG, et al. A comparison of measures of disproportionality for signal detection in spontaneous reporting systems for adverse drug reactions. Pharmacoepidemiol Drug Saf. 2002; 11: 3-10.

16. Bate A, Lindquist M, Edwards IR, et al. A Bayesian neural network method for adverse drug reaction signal generation. Eur J Clin Pharmacol. 1998; 54: 315-321.

17. Sakaeda T, Tamon A, Kadoyama K, et al. Data mining of the public version of the FDA Adverse Event Reporting System. Int J Med Sci. 2013; 10: 796-803.

18. Almenoff JS, Pattishall EN, Gibbs TG, et al. Novel statistical tools for monitoring the safety of marketed drugs. Clin Pharmacol Ther. 2007; 82: 157-166.

19. Hallas J. Evidence of depression provoked by cardiovascular medication: a prescription sequence symmetry analysis. Epidemiology. 1996; 7: 478-484.

20. Tsiropoulos I, Andersen M, Hallas J. Adverse events with use of antiepileptic drugs: a prescription and event symmetry analysis. Pharmacoepidemiol Drug Saf. 2009; 18: 483-491.

21. Hallas J, Gaist D, Bjerrum L. The waiting time distribution as a graphical approach to epidemiologic measures of drug utilization. Epidemiology. 1997; 8: 666-670.

22. Morris JA, Gardner MJ. Calculating confidence intervals for relative risks (odds ratios) and standardised ratios and rates. Br Med J (Clin Res Ed). 1988; 296: 1313-1316.

23. Imfeld $\mathrm{P}$, Bodmer M, Jick SS, et al. Benzodiazepine use and risk of developing alzheimer's disease or vascular dementia: a case-control analysis. Drug Saf. 2015.

24. Billioti de Gage S, Moride Y, Ducruet T, et al. Benzodiazepine use and risk of Alzheimer's disease: case-control study. BMJ. 2014; 349: g5205.

25. Taragano FE, Allegri RF, Krupitzki H, et al. Mild behavioral impairment and risk of dementia: a prospective cohort study of 358 patients. J Clin Psychiatry. 2009; 70: 584-592.

26. Lyketsos CG, Lopez O, Jones B, et al. Prevalence of neuropsychiatric symptoms in dementia and mild cognitive impairment: results from the cardiovascular health study. JAMA. 2002; 288: 1475-1483.

27. Maclure M, Fireman B, Nelson JC, et al. When should case-only designs be used for safety monitoring of medical products? Pharmacoepidemiol Drug Saf. 2012; 21 Suppl 1: 50-61.

28. Takada $\mathrm{M}$, Fujimoto $\mathrm{M}$, Motomura $\mathrm{H}$, et al. Inverse association between sodium channel-blocking antiepileptic drug use and cancer: data mining of spontaneous reporting and claims databases. Int J Med Sci. 2016; 13: 48-59.

29. Wahab IA, Pratt NL, Wiese MD, et al. The validity of sequence symmetry analysis (SSA) for adverse drug reaction signal detection. Pharmacoepidemiol Drug Saf. 2013; 22: 496-502.

30. Wahab IA, Pratt NL, Kalisch LM, et al. Sequence symmetry analysis and disproportionality analyses: what percentage of adverse drug reaction do they signal? Advances in Pharmacoepidemiology \& Drug Safety. 2013; 2.

31. Tannenbaum C, Paquette A, Hilmer S, et al. A systematic review of amnestic and non-amnestic mild cognitive impairment induced by anticholinergic, antihistamine, GABAergic and opioid drugs. Drugs Aging. 2012; 29: 639-658.

32. Curran HV. Tranquillising memories: a review of the effects of benzodiazepines on human memory. Biol Psychol. 1986; 23: 179-213.

33. Petersen RC, Doody R, Kurz A, et al. Current concepts in mild cognitive impairment. Arch Neurol. 2001; 58: 1985-1992.

34. Busse A, Hensel A, Guhne U, et al. Mild cognitive impairment: long-term course of four clinical subtypes. Neurology. 2006; 67: 2176-2185.

35. Hutchinson MA, Smith PF, Darlington CL. The behavioural and neuronal effects of the chronic administration of benzodiazepine anxiolytic and hypnotic drugs. Prog Neurobiol. 1996; 49: 73-97.

36. Shimohama $\mathrm{S}$, Taniguchi $\mathrm{T}$, Fujiwara $\mathrm{M}$, et al. Changes in benzodiazepine receptors in Alzheimer-type dementia. Ann Neurol. 1988; 23: 404-406. 